\title{
Nonlinear Ion Dynamics in Hall thruster plasma source by Ion Transit-time Instability
}

\author{
Youbong Lim, ${ }^{1}$ Wonho Choe ${ }^{1, a)}$, Stéphane Mazouffre ${ }^{2}$, Jae Sun Park, ${ }^{1}$ Holak Kim, ${ }^{1}$ Jongho Seon, ${ }^{3}$ and L. \\ Garrigues $^{4,5}$ \\ ${ }^{1}$ Department of Physics, Korea Advanced Institute of Science and Technology, 291 Daehak-ro, Yuseong-gu, \\ Daejeon 34141, Republic of Korea \\ ${ }^{2}$ ICARE-CNRS, University of Orléans, 1c Av. de la Recherche Scientifique, 45071 Orléans, France \\ ${ }^{3}$ Department of Space Science and Astronomy, Kyung Hee University, 1732 Deogyeong-daero, Giheung-gu, \\ Yongin-si, Gyeonggi-do 17104, Republic of Korea \\ ${ }^{4}$ Laplace, Université de Toulouse, UPS, INPT Toulouse, France \\ ${ }^{5}$ CNRS, Laplace, Université Paul Sabatier 118, route de Narbonne 31062 Toulouse Cedex 9, France \\ ${ }^{a)}$ Author to whom correspondence should be addressed. Electronic mail: wchoe@kaist.ac.kr
}

Key words: crossed-field discharge, high-energy tail, IEDF, ion transit-time instability, nonlinear ion dynamics

High-energy tail formation in an ion energy distribution function (IEDF) is explained in a Hall thruster plasma with the stationary crossed electric and magnetic fields whose discharge current is oscillated at the ion transit-time scale with a frequency of $360 \mathrm{kHz}$. Among ions in different charge states, singly charged $\mathrm{Xe}$ ions $\left(\mathrm{Xe}^{+}\right)$have an IEDF that is significantly broadened and shifted toward the high-energy side, which contributes to tail formation in the entire IEDF. Analytical and numerical investigations confirm that the IEDF tail is due to nonlinear ion dynamics in the ion transit-time oscillation. 
The formation of a high-energy tail in an ion energy distribution function (IEDF) has been observed during wave heating in magnetized fusion plasma soruces, which is explained by nonlinear or stochastic ion acceleration [1-4]. High-energy ion tail formation has also been observed in Hall thruster plasmas in which crossed stationary axial electric and radial magnetic fields are provided to produce plasmas with azimuthal Hall drift electrons and unmagnetized ions due to their large Larmor radii [5-8]. A Hall thruster plasma soruce has various oscillation modes in a wide frequency spectrum, from a few kilohertz to tens of megahertz, and each oscillation mode is enhanced by the plasma instability that is closely dependent upon operating conditions such as the mass flow rate of the gas, anode voltage, and magnetic field. Ion transittime instability in Hall thruster plasma was reported as being triggered by a longitudinal acoustic wave in the $100-500 \mathrm{kHz}$ frequency range [9-12]. Electric field oscillation at the ion transit-time scale was observed to have a relationship with the tail ion formation, which can be explained by a proposed wave-riding mechanism that suggests the existence of a relationship between the fast ion generation and electric field oscillation [5,6]. Despite many experiments and numerical simulations showing the existence of the high-energy tail and ion transit-time instability, the exact nature of the tail formation is still elusive.

The Hall thruster plasma studied in this work was produced by a cylindrical type Hall thruster that has an open-ended discharge chamber consisting of a dielectric channel of $24 \mathrm{~mm}$ in length and $50 \mathrm{~mm}$ in diameter. The magnetic field is provided by a pair of electromagnets and a magnetic circuit. In the cylindrical type Hall thruster, the central magnetic core of the conventional annular type thruster is removed to increase the volume-to-surface ratio of the plasma [13]. It is noted that many interesting physical processes that are not shown in conventional annular thrusters take place in cylindrical thruster plasmas, including a high fraction of multiply charged ions [14,15]. The detailed description of the experimental set-up is found in Refs. [14-18]. Throughout the experiment, an electric potential of $300 \mathrm{~V}$ was applied to the anode and the mass flow rate of Xe gas supplied through the anode was either 4.0 or 10.0 sccm.

The plasma oscillation was experimentally characterized by analyzing the discharge current and it was Fourier-transformed to obtain spectral distributions as plotted in Figs. 1(a) and (c). The plasma oscillations occurring at low frequencies $(<50 \mathrm{kHz})$ are the breathing mode or ionization oscillation caused by periodic depletion of neutral particles due to ionization. The period of this oscillation is on the order of the transit-time of the neutrals supplied to the discharge region [7]. On the contrary, a separate large peak at $360 \mathrm{kHz}$ appears at $10.0 \mathrm{sccm}$ as shown in Fig. 1(b). Its oscillation period is on the order of the transit-time of the ions passing through the acceleration region; hence, it is referred to as the ion transit-time oscillation $[9,10]$. The oscillation was also observed in the kilowatt class conventional annular type Hall thruster plasmas in the $150-600 \mathrm{kHz}$ range [5,6]. Although numerical simulations predicted oscillations at a few $100 \mathrm{kHz}$ [9-11], the origin of this oscillation is still unclear. It is noteworthy that the transit-time oscillation has neither been previously observed nor reported in cylindrical Hall thruster plasmas.

The energy distribution functions of the ions with different charge states were obtained using an ExB probe. Since the energy resolution of the probe depends on the energy and charge state of the incoming ion, the analysis of the measured probe spectra becomes complicated, 
and we developed a mathematical method to reconstruct IEDFs from the measured probe spectra. Both the detailed probe configuration and IEDF reconstruction method are described in our previous study [8]. Figures 1(c) and (d) show the IEDFs for the plasmas whose oscillation frequency spectra of the discharge current are depicted in Fig. 1(a) and (b), respectively. The red curves indicate the IEDF for $\mathrm{Xe}^{+}$ions, and the black curves are for $\mathrm{Xe}^{2+}$ and $\mathrm{Xe}^{3+}$ ions. The entire IEDFs, obtained by adding the IEDFs for $\mathrm{Xe}^{+}, \mathrm{Xe}^{2+}$, and $\mathrm{Xe}^{3+}$ ions, are denoted by the blue curves. From the figure, a few findings are made: first, at $4.0 \mathrm{sccm}$, both the $\mathrm{Xe}^{+}$IEDF and the entire IEDF are roughly symmetric with respect to their peaks; second, at $10 \mathrm{sccm}$, the peak energy of the $\mathrm{Xe}^{+}$IEDF is higher than that of the entire IEDF, which significantly distorts the entire IEDF to form a high-energy tail. It is important to emphasize that the tail formation beyond the anode voltage is mainly due to $\mathrm{Xe}^{+}$ions. Moreover, Fig. 1 demonstrates that the formation of a high-energy tail in the entire IEDF is closely related to the appearance of the oscillation at $360 \mathrm{kHz}$, which was also confirmed under other discharge conditions in our experiment.

The correlation between the IEDF tail formation and plasma oscillation was analytically investigated by considering the dynamics of an ion in the presence of the oscillating plasma potential. Here, the plasma oscillation is taken into account by defining a time-dependent onedimensional plasma potential and its corresponding electric field which oscillates with a frequency $\omega$, as depicted in Figs. 2(a) and (b), respectively. The ions are assumed to have a zero initial velocity and to be collisionless because the acceleration region is shorter than the ion-neutral collisional mean free path. In order to investigate the frequency dependence of the ion dynamics, the electric field is assumed to oscillate harmonically as

$$
\begin{aligned}
& E(z, t)=E\left(z-z_{c}(t)\right), \\
& z_{c}(t)=\delta \cos (\omega t+\varphi),
\end{aligned}
$$

where $t$ is the time, $z$ is the spatial coordinate along the thruster axis with $z=0$ at the anode, $\delta$ is the oscillation amplitude that is much smaller than the characteristic length of the acceleration zone $L$, and the phase $\varphi$ is the initial phase of a test ion to be accelerated by the electric field. The spatial profile of the electric field can be arbitrarily given but it vanishes outside the acceleration zone. As described in Eq. (1), the electric field is set to oscillate in the $z$ direction, and the electric potential and field profiles in Fig. 2 are not restricted to the curves depicted in the figure (which is Gaussian), which are presented for the sake of better understanding.

Since $\delta \ll L$, the equation of motion of an ion is approximated as

$$
\frac{m}{q} \frac{d v}{d t}=E(z, t) \simeq \widehat{E}(z)-\frac{d \hat{E}(z)}{d z} z_{c}(t)
$$

where $\hat{E}(z)$ is the time-independent spatial function of the electric field which satisfies a Dirichlet boundary condition of $\widehat{E}(z \leq 0)=\hat{E}(z \geq L)=0$, and $m, q$, and $v$ are the mass, charge, and speed of the ion, respectively. If the speed of the ion is set as $\boldsymbol{v} \simeq \boldsymbol{v}_{\mathbf{0}}+$ $\epsilon v_{1}$ where $v_{0}$ and $v_{1}$ are the unperturbed and the first order perturbed solution 
with the expansion parameter of $\epsilon=\delta / L \ll 1$, respectively, then Eq. (3) becomes

$$
\frac{m}{q} \frac{d v_{0}}{d t}+\epsilon \frac{m}{q} \frac{d v_{1}}{d t}=\widehat{E}(z)-\hat{E}^{\prime}(z) \frac{\delta}{L} L \cos (\omega t+\varphi)
$$

where $\hat{E}^{\prime}(z)=d \hat{E}(z) / d z$. The unperturbed part of Eq. (4) corresponding to the timeindependent electric field and its solution are then given as

$$
\begin{gathered}
\frac{m}{q} \frac{d v_{0}}{d t}=\hat{E}(z), \\
v_{0}(z)=\left\{\frac{2 q}{m} \int_{0}^{z} \hat{E}\left(z^{\prime}\right) d z^{\prime}\right\}^{1 / 2},
\end{gathered}
$$

where $d z_{0} / d t=v_{0}$, and $v_{0}(z)$ for an arbitrarily given $\hat{E}(z)$ is depicted in Fig. 2(b) as the blue curve. On the other hand, the equation for $v_{1}$ from Eq. (4) becomes

$$
\frac{m}{q} \frac{d v_{1}}{d t}=-\hat{E}^{\prime}(z) L \cos (\omega t+\varphi) \simeq-\hat{E}^{\prime}\left(z_{0}(t)\right) L \cos (\omega t+\varphi),
$$

The right-hand side of Eq. (7) is defined on the $z$ coordinate, and now it is transformed into the time coordinate by using the unperturbed solution $z_{0}(t)$. Let us define

$$
\alpha(t)=-\frac{q L}{m} \hat{E}^{\prime}\left(z_{0}(t)\right)
$$

then, $\alpha(t)$ can be considered as the effective ion acceleration that mainly results from the spatial derivative of the electric field and the unperturbed solution. The perturbed solution $v_{1}$ is solved as

$$
v_{1}(t)=\int_{0}^{t} \alpha\left(t^{\prime}\right) \cos \left(\omega t^{\prime}+\varphi\right) d t^{\prime}=\operatorname{Re}\left[e^{i \varphi} \int_{0}^{t} e^{i \omega t \prime} \alpha\left(t^{\prime}\right) d t^{\prime}\right]
$$

Since $z_{0}(t)$ is a monotonically increasing function with respect to time, $\alpha(t)$ satisfies the same Dirichlet boundary conditions as the electric field, i.e., $\alpha(t \leq 0)=\alpha(t \geq \tau)=0$, where $\tau$ is the ion transit-time or the time for the ion traversing the acceleration zone. So, the integration limit can be extended to infinity, with which we have

$$
\begin{aligned}
& v_{1}(\tau)=\operatorname{Re}\left[e^{i \varphi} \mathrm{A}(\omega)\right], \\
& \mathrm{A}(\omega)=\int_{-\infty}^{\infty} e^{i \omega t} \alpha(t) d t .
\end{aligned}
$$

Equation (10) indicates that the perturbed solution $v_{1}$ is the Fourier transform of $\alpha(t)$, which becomes significant with respect to $v_{0}$ when the oscillation time scale of the electric field $2 \pi / \omega$ is comparable with $\tau$. In the case depicted in Fig. 2(c), $v_{1}(\tau) \sim 0.5 v_{0}(\tau)$. As presented in Eq. (6), $v_{0}(\tau)$ is uniquely given by the anode voltage since the ion is assumed to 
be initially positioned at the anode, but $v_{1}$ is determined not only by $\alpha$ but also by the initial phase $\varphi$, so that $v_{1}$ can be positive or negative depending on $\varphi$. On the other hand, at very high $\omega$ where $\omega \tau \gg 1, v_{1}$ decays significantly as time approaches $\tau$ or as the ion is accelerated through the acceleration zone, i.e. $v_{1}(\tau) \ll v_{0}(\tau)$. In the case of Fig. 2(d) where $\omega \tau \gg 1, v_{1}(\tau) \sim 0.01 v_{0}(\tau)$. On the other hand, for $\omega \tau \ll 1$, the oscillating electric field may be regarded as a static field to the ions, which makes $v_{1}(\tau)$ negligible. The aforementioned results can be summarized as follows: i) $v_{1}$ is determined by the spatial derivative of the electric field; ii) $v_{1}(\tau)$ may become large when the oscillation time scale is comparable with the ion transit-time $(\omega \tau \sim 1)$; and iii) the large $v_{1}(\tau)$ due to the acceleration further by the oscillating electric field can lead to an ion energy higher than $q V_{a}$ ( $q$ and $V_{a}$ being the ion charge and anode voltage, respectively) and IEDF broadening.

In order to quantitatively examine the ion dynamics, the equation of motion given in Eq. (3) was numerically solved for each singly, doubly, and triply charged Xe ion under the electric field shown in Fig. 2 oscillating with $\omega / 2 \pi$ varied from $10 \mathrm{kHz}$ to $10 \mathrm{MHz}$. The same procedure was repeated for one thousand independent ions with a randomly given initial phase $\varphi$. Whether an ion gains or loses energy by the oscillating electric field is determined by the initial phase $\varphi$, however the amount of energy gained or lost depends on the frequency of the oscillating field $\omega$ for a given ion energy (i.e. speed). Therefore, the ion energy spread depends strongly on the oscillation frequency and the ion charge.

Shown in Fig. 3 are the time-averaged energy distributions for the $\mathrm{Xe}^{+}, \mathrm{Xe}^{2+}$, and $\mathrm{Xe}^{3+}$ ions where the abscissa and ordinate are $\omega$ and the ion energy per charge, respectively. The figure shows that the distributions broaden from approximately $100 \mathrm{kHz}$ and the broadening width becomes significantly increased in the $100 \mathrm{kHz}-5 \mathrm{MHz}$ range. It is noted that this energy broadening results from the perturbed solution $v_{1}(\tau)$ due to the oscillating electric field since the unperturbed solution $v_{0}(\tau)$ is determined uniquely by the anode voltage. In the presence of the electric field oscillating at either lower than $100 \mathrm{kHz}$ or higher than $5 \mathrm{MHz}$, the calculated ion energy per charge $W / q$ for all $\mathrm{Xe}^{+}, \mathrm{Xe}^{2+}$, and $\mathrm{Xe}^{3+}$ ions are approximately equal to $V_{a}$ ( $V_{a}=300 \mathrm{~V}$ in this case) where $W$ is the ion energy and $q$ is the ion charge. It implies that $v_{1}$ is negligible so that $v \simeq v_{0}$ at these frequencies, i.e., the oscillating electric field gives negligible alterations to the ion dynamics. With the electric field oscillating at 100 $\mathrm{kHz}-5 \mathrm{MHz}$, however, the ion energy becomes remarkably broadened due to the large $v_{1}$. The presence of ions with $W / q$ higher than $V_{a}$ is due to the fact that the acceleration motion is positively synchronized with the oscillating electric field owing to the initial phase condition of the ions. At the measured oscillation frequency of $360 \mathrm{kHz}$, the peak $W / q$ values (denoted as the blackish curve in each energy band) correspond to 380, 320, and $300 \mathrm{~V}$ for $\mathrm{Xe}^{+}, \mathrm{Xe}^{2+}$, and $\mathrm{Xe}^{3+}$ ions, respectively. The average $W / q$ values of each ion are 347,319 , and $306 \mathrm{~V}$, respectively, which are higher than $V_{a}$. These values indicate that the largest contribution to the high-energy ion tail formation is expected from $\mathrm{Xe}^{+}$ions under the same oscillating electric field, and this result is consistent with the measurement shown in Fig. 1. The reason why the largest contribution is from $\mathrm{Xe}^{+}$ions is that ions with higher charge states spend less time inside the discharge channel, i.e. shorter transit-time, which requires higher oscillation frequency for the ions to be synchronized. It is emphasized that the ions gain kinetic energy higher than the 
applied anode voltage, and this nonlinear behavior results from the resonance phenomenon whereby the ions see the static electric field under the condition, $\omega / k \approx v$. The resonant ion velocity $v$ and its kinetic energy $W=m v^{2} / 2$ are determined not only by the anode voltage but also by $\omega$ of the oscillating electric field via the wave riding mechanism, as demonstrated in Ref. [5]. The average $W / q$ can be higher than $V_{a}$ if $\omega$ is produced in such a way that $W=m v^{2} / 2=m(\omega / k)^{2} / 2>q V_{a}$, or $\omega>\left(2 q V_{a} / m_{i}\right)^{1 / 2} k$. it is noteworthy that the existence of ions with energy higher than the anode voltage was experimentally observed in previous works [8,16-20].

In the following, understanding of the implication of the electric field oscillation in the time domain is attempted from the same calculation. Shown in Fig. 4 are the ion currents for different charge species obtained by calculating an ion motion and counting the number of ions that arrive at an imaginary ion collector located at the same location as the Faraday probe in the experiment, which is $\mathbf{0 . 5} \mathbf{~}$ away from the thruster exit. While Fig. 3 presents the time-averaged ion energy broadening, Fig. 4 plots the temporal values of ion current and $W / q$ of each charge state ion under the electric field oscillating at $360 \mathrm{kHz}$. This figure shows that the peak current of the higher charge state ion appears earlier in time than the lower charge state ions due to their shorter transit-time inside the discharge channel. Supposed that there is a charge collector located next to the acceleration zone, the ratio of the collected currents will depend on time. For instance, at half a period of the oscillation, the order of the current values at the collector is $\mathrm{Xe}^{+}, \mathrm{Xe}^{3+}$, and $\mathrm{Xe}^{2+}$ ions. In addition, the ion energy per charge also depends on time due to the oscillating electric field as shown in Fig. 4(b). At 1/10 period, the $\mathrm{Xe}^{3+}$ ions with $W / q$ of $305 \mathrm{~V}$ and $345 \mathrm{~V}$ reach the collector. However, at $1 / 2$ period, all three different charge state ions with different energies are collected, i.e., 337, $384 \mathrm{~V} \mathrm{Xe}^{+}$ions, 261, $313 \mathrm{~V} \mathrm{Xe}^{2+}$ ions, and 252, $285 \mathrm{~V} \mathrm{Xe}^{3+}$ ions. These different constituents with different population and energy per charge bring about the broadening and shift of the energy distributions, ultimately leading to the formation of tail ions in the IEDF. We believe that the ion current oscillation presented in Fig. 4(a) gives significant feedback to the discharge current, which enhances the ion transit-time plasma oscillation. Since the population of singly charged ions is dominant in the thruster plasma $[14,15]$, the plasma oscillation synchronized with $\mathrm{Xe}^{+}$ ions is dominantly enhanced.

In conclusion, the high-energy tail in an IEDF was observed only when the ion transittime instability was enhanced in the thruster plasma, which gives rise to a shift of the $\mathrm{Xe}^{+}$peak toward the high-energy side and tail formation in the entire IEDF. The nonlinear ion dynamics was analytically and numerically investigated to confirm the strong correlation between the tail formation and ion transit-time plasma oscillation. The results show that the ion velocity is determined not only by the charge and electric field profile but also by the variation of the electric field with time. The ion velocity $v_{1}$, strongly correlated to the field oscillation, becomes extremely important when the oscillation time scale is comparable with the ion transittime ( $\omega \tau \sim 1)$, and $v_{1}$ is responsible for the tail formation in an IEDF.

This work was partly supported by the Space Core Technology Program (Grant No. 2014M1A3A3A02034510) through the National Research Foundation of Korea funded by Ministry of Science, ICT and Future Planning, and also partly supported by the Korea Institute of Materials Science (KIMS) (Grant No. 10043470) funded by the Ministry of Trade, Industry 
and Energy of Korea and the National Research Foundation of Korea (NRF), which was funded by the Ministry of Science, ICT (No. NRF-2014M1A7A1A03045089). 


\section{References}

[1] G. R. Smith and A. N. Kaufman, Phys. Rev. Lett. 34, 26 (1975).

[2] A. Fukuyama, H. Momota, and R. Itatani, Phys. Rev. Lett. 38, 13 (1977).

[3] F. Skiff, F. Anderegg, and M. Q. Tran, Phys. Rev. Lett. 58, 14 (1987).

[4] B. Jorns and E. Y. Choueiri, Phys. Rev. Lett. 106, 085002 (2011).

[5] S. Mazouffre, V. Kulaev, J. Pérez-Luna, Plasma Sources Sci. Technol. 18, 034022 (2009).

[6] J. Vaudolon, and S. Mazouffre, Plasma Sources Sci. Technol. 24, 032003 (2015).

[7] E. Y. Choueiri, Phys. Plasmas 8, 4 (2001).

[8] Y. Lim, H. Kim, W. Choe, S. H. Lee, J. Seon, and H. J. Lee, Phys. Plasmas 21, 103502 (2014).

[9] S. Barral, K. Makowski, Z. Peradzyski, and M. Dudeck, Phys. Plasmas 12, 073504 (2005).

[10] J. Bareilles, G. J. M. Hagelaar, L. Garrigues, C. Boniface, J. P. Boeuf, and N. Gascon, Phys. Plasmas 11, 3035 (2004).

[11] Y. Esipchuck, A. Morozov, G. Tilinin, and A. Trofimov, Sov. Phys. Tech. Phys. 18, 928 (1974).

[12] Y. Esipchuck and G. Tilinin, Sov. Phys. Tech. Phys. 21, 417 (1976).

[13] Y. Raitses, N. J. Fisch, Phys. Plasmas 8, 2579 (2001).

[14] H. Kim, Y. Lim, W. Choe, and J. Seon, Appl. Phys. Lett. 105, 144104 (2014).

[15] H. Kim, Y. Lim, W. Choe, S. Park, and J. Seon, Appl. Phys. Lett. 106, 154103 (2015).

[16] M. Seo, J. Lee, J. Seon, H. J. Lee, and W. Choe, Phys. Plasmas 20, 023507 (2013).

[17] M. Seo, J. Lee, J. Seon, H. J. Lee, and W. Choe, Appl. Phys. Lett. 103, 133501 (2013).

[18] J. Lee, M. Seo, J. Seon, H. J. Lee, and W. Choe, Appl. Phys. Lett. 99, 131505 (2011).

[19] K. D. Diamant, et al., IEEE Trans. Plasma Sci. 38, 1052 (2010).

[20] A. Smirnov, Y. Raitses, and N. J. Fisch, Phys. Plasmas 14, 057106 (2007). 


\section{FIGURE CAPTIONS}

FIGURE 1. (a) Spectral distribution of the discharge current oscillations and (c) the corresponding IEDFs of $\mathrm{Xe}^{+}$(red), $\mathrm{Xe}^{2+}$ (black), and $\mathrm{Xe}^{3+}$ ions (black dashed), and the entire IEDF (blue) at $4.0 \mathrm{sccm}$ Xe flow rate. (b) and (d) are those for $10.0 \mathrm{sccm}$. Measurements were performed at $600 \mathrm{~mm}$ from the thruster exit.

FIGURE 2. (a) Oscillating plasma potential, (b) oscillating electric field profile (black curves) and the unperturbed solution $v_{0}$ (blue curve), (c) the effective ion acceleration $\alpha$ (black curve) and the perturbed solution $v_{1}$ (blue curve) when $\omega \tau / 2 \pi=2.5$, and (d) when $\omega \tau / 2 \pi=24$.

FIGURE 3. Ion energy distributions numerically calculated with $\omega / 2 \pi=10 \mathrm{kHz}-10 \mathrm{MHz}$ for (a) $\mathrm{Xe}^{+}$, (b) $\mathrm{Xe}^{2+}$, and (c) $\mathrm{Xe}^{3+}$ ions. The anode voltage was set to be $300 \mathrm{~V}$, as in the experimental conditions.

FIGURE 4. (a) Normalized ion current, (b) ion energy per charge calculated for $\mathrm{Xe}^{+}$(red), $\mathrm{Xe}^{2+}$ (blue), and $\mathrm{Xe}^{3+}$ (black) as a function of time in one oscillation period. These plots are for the oscillation frequency of $360 \mathrm{kHz}$. 

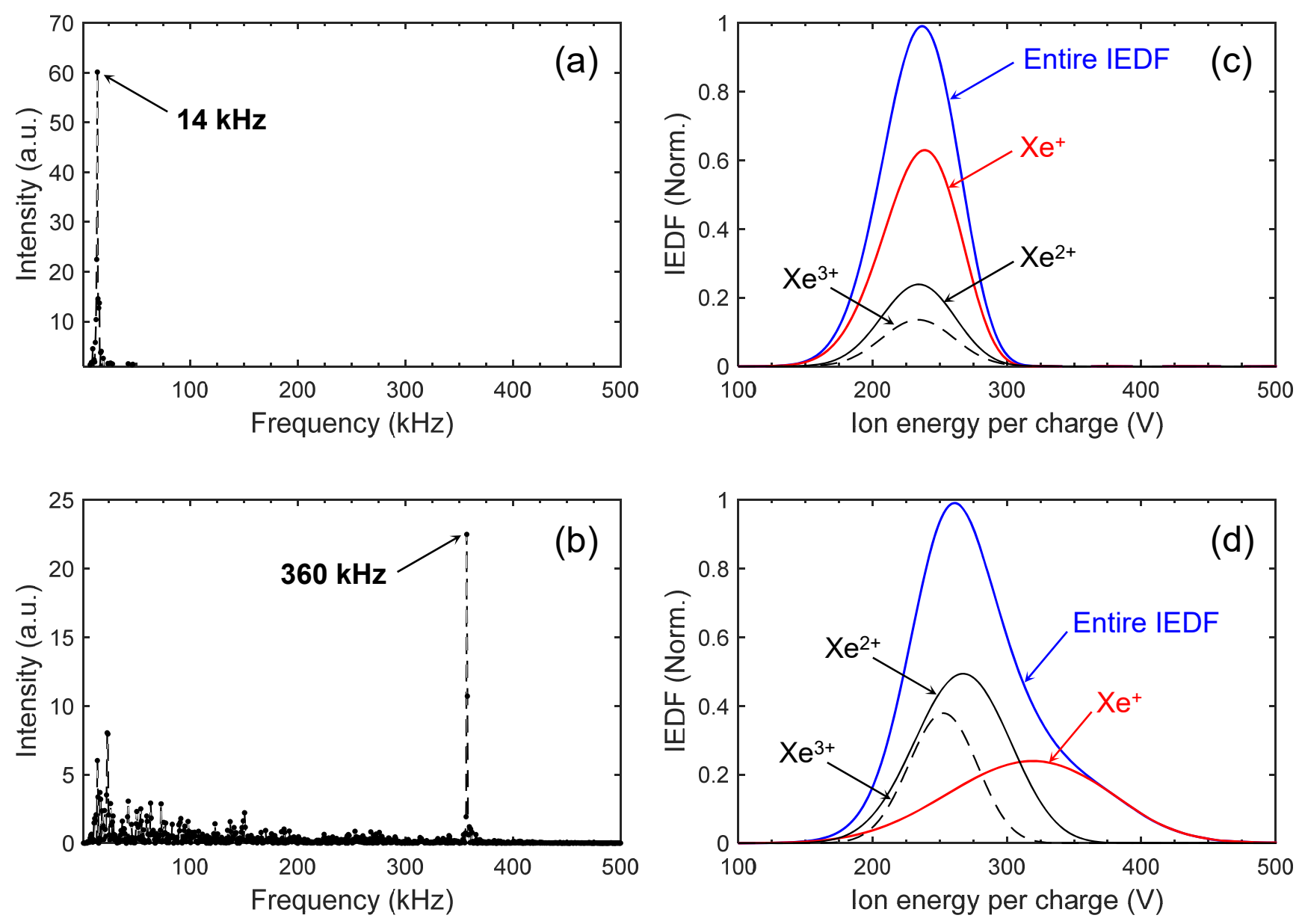

Figure 1. Lim et al. 
(a)

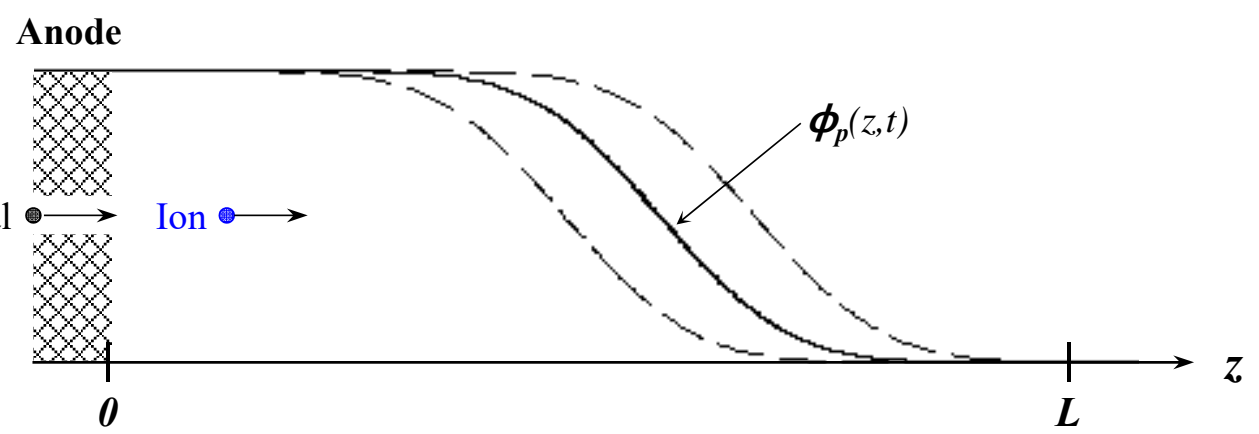

(b)

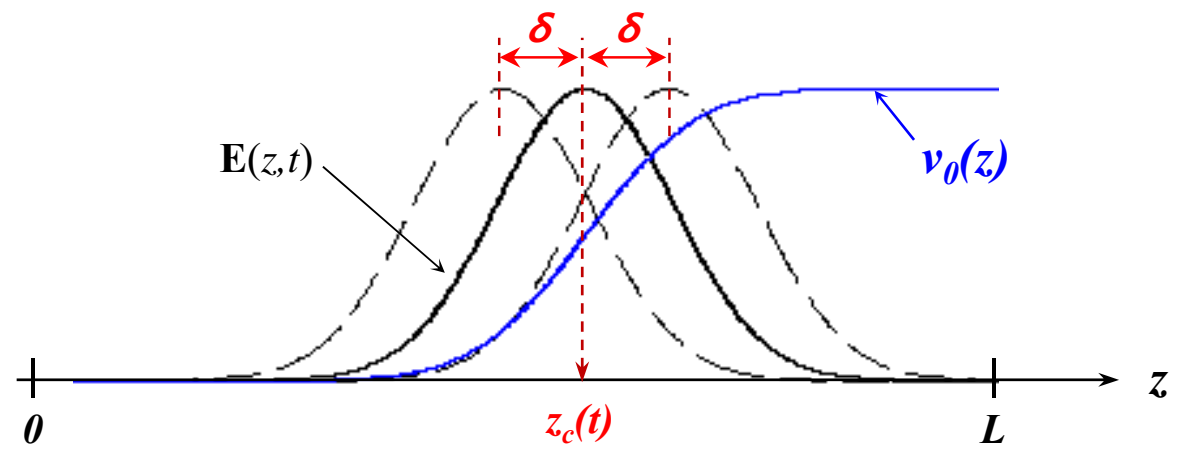

(c)

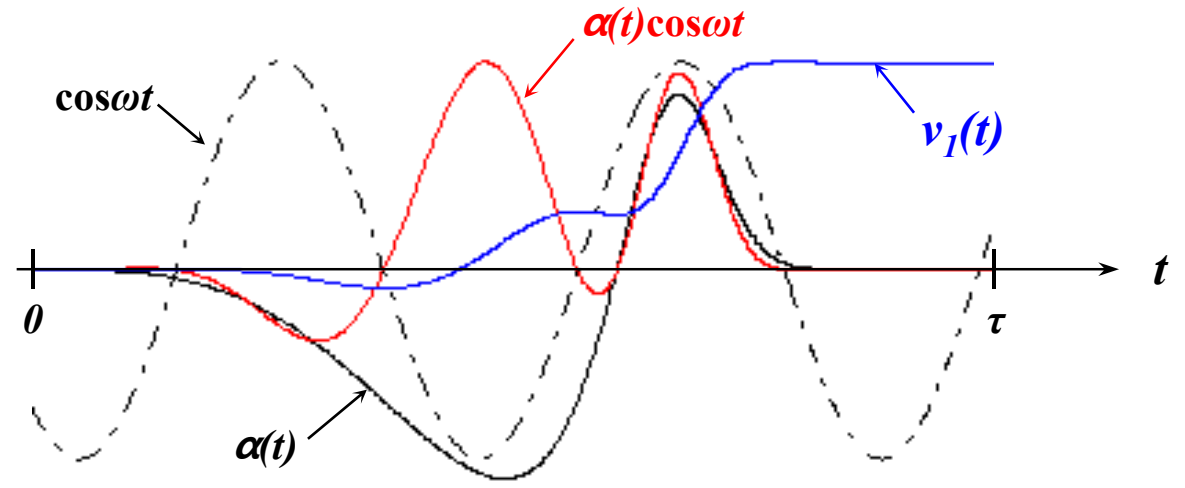

(d)

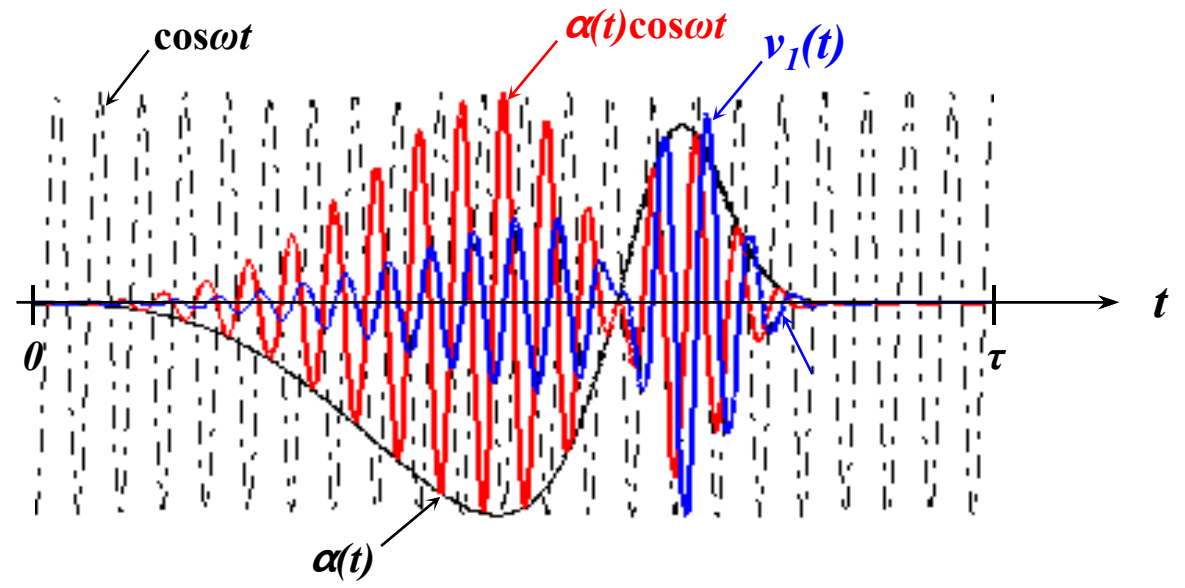

Figure 2. Lim et al. 

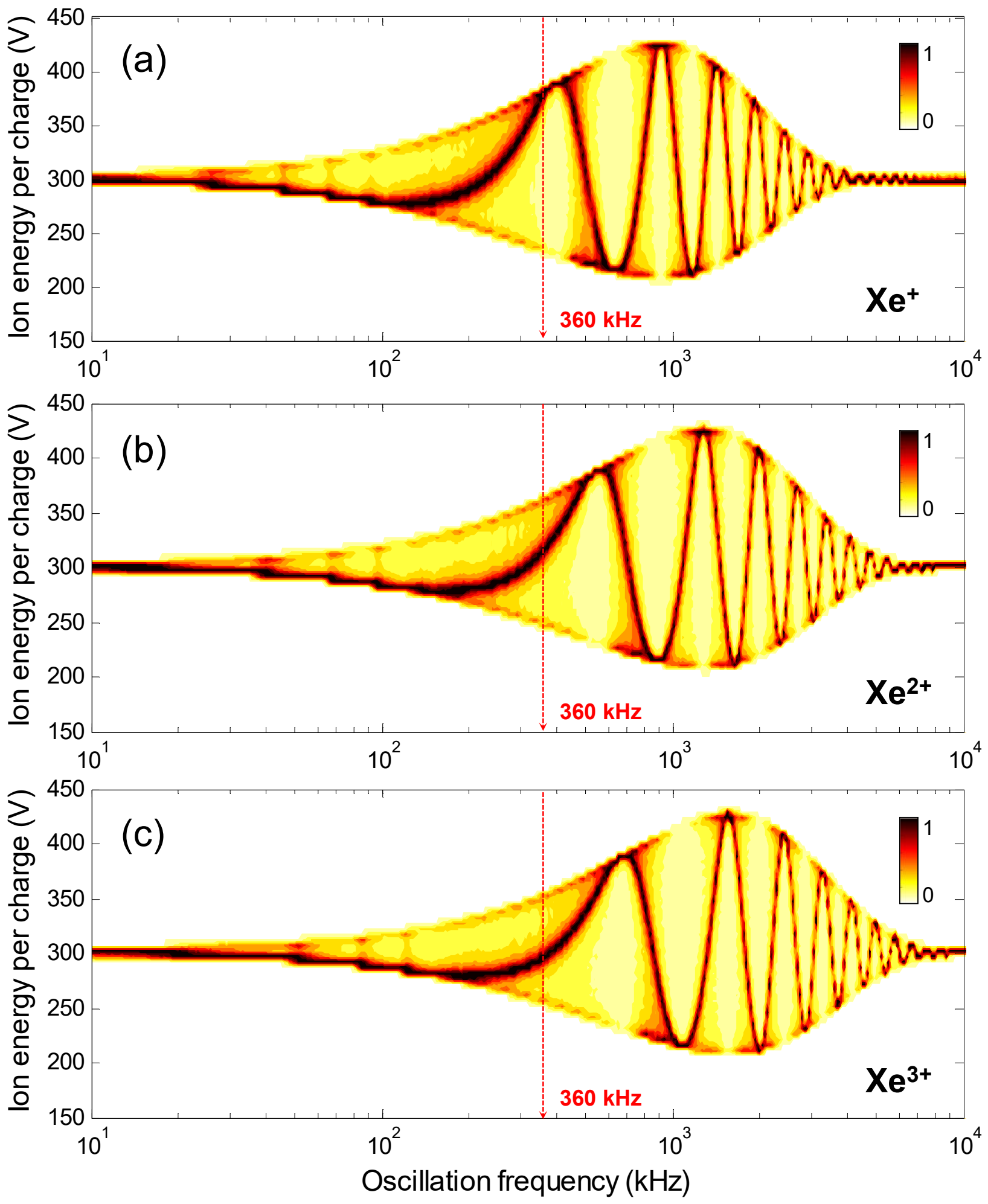

Figure 3. Lim et al. 

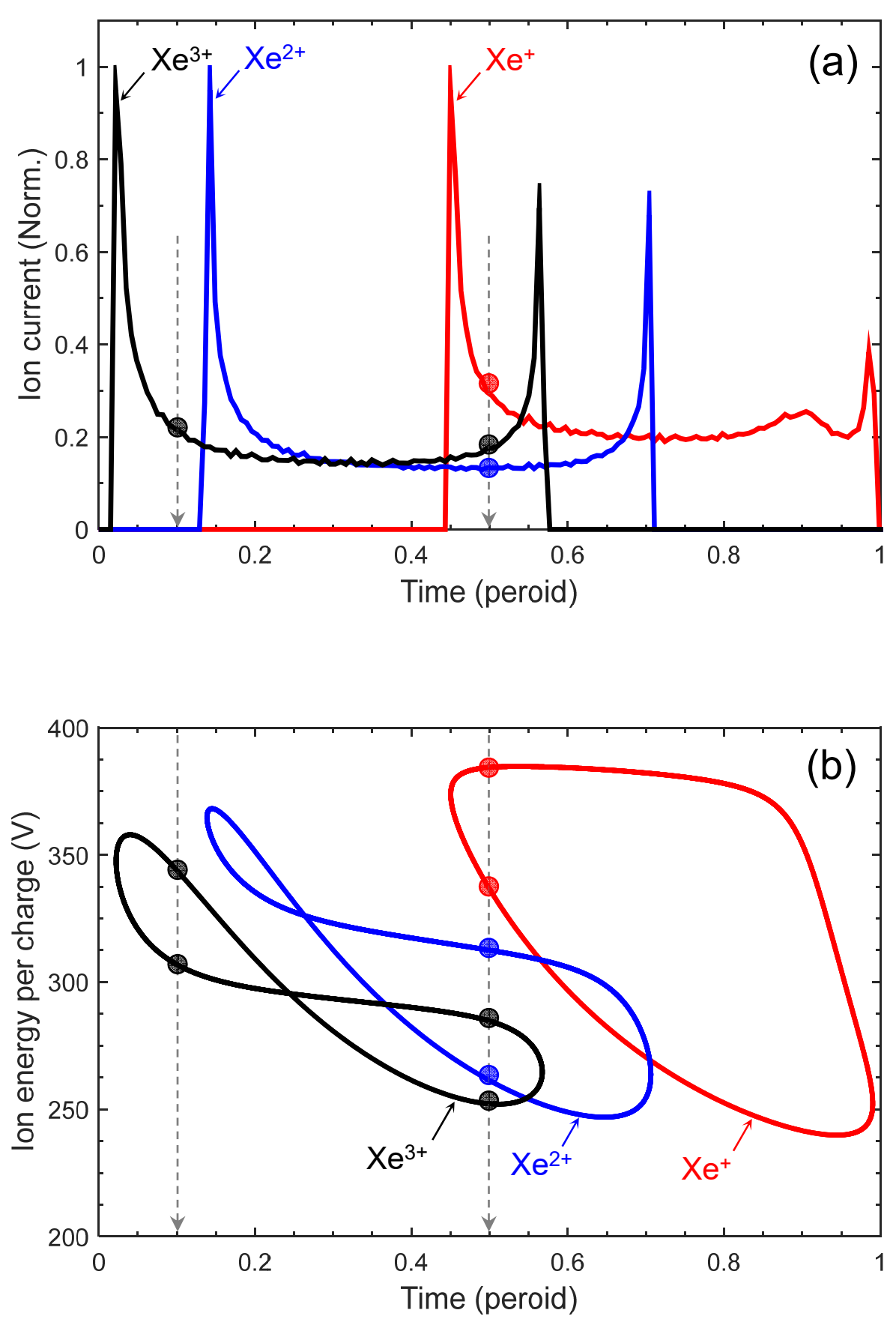

Figure 4. Lim et al. 